\title{
Comparison of Iteration Schemes for the Solution of the Multidimensional Schrödinger-Poisson Equations
}

\author{
A. TRELLAKIS ${ }^{\mathrm{a}, *}$, A. T. GALICK ${ }^{\mathrm{a}}$, A. PACELLI $^{\mathrm{b}}$ and U. RAVAIOLI ${ }^{\mathrm{a}}$ \\ ${ }^{a}$ Beckman Institute, University of Illinois at Urbana-Champaign,Urbana, IL 61801; \\ ${ }^{\mathrm{b}}$ Dipartimento di Elettronica e Informazione and CEQSE-CNR, Politecnico di Milano, \\ Piazza Leonardo da Vinci 32, 20133 Milano, Italy
}

\begin{abstract}
We present a fast and robust iterative method for obtaining self-consistent solutions to the coupled system of Schrödinger's and Poisson's equations in quantum structures. A simple expression describing the dependence of the quantum electron density on the electrostatic potential is used to implement a predictor-corrector type iteration scheme for the solution of the coupled system of differential equations. This approach simplifies the software implementation of the nonlinear problem, and provides excellent convergence speed and stability. We demonstrate the algorithm by presenting an example for the calculation of the two-dimensional bound electron states within the cross-section of a GaAs-AlGaAs based quantum wire. For this example, six times fewer iterations are needed when our predictorcorrector approach is applied, compared to a corresponding underrelaxation algorithm.
\end{abstract}

Keywords: Semiconductor, simulation, quantum wire, iteration, predictor-corrector, Schrödinger-Poisson

\section{INTRODUCTION}

As electronic device dimensions approach nanometer scale, quantum effects are expected to dominate their electronic properties. Consequently there is considerable interest in the efficient numerical simulation of such structures, not only for exploring novel device architectures but also for maintaining reliability for reduced present-day devices. In this paper we present a fast and robust iterative method for solving the two-dimensional
Schrödinger-Poisson equations and obtaining the electron states in the cross-section of a quantum wire, and we compare the efficiency of our method with the standard underrelaxation algorithm.

The physical model used to describe the quantum wire consists, in the effective mass approximation, of Schrödinger's equation

$$
-\frac{\hbar^{2}}{2} \nabla\left[\frac{1}{m^{*}} \nabla \psi_{l}\right]+\left[V_{h}-e \phi+V_{\mathrm{xc}}(n)-E_{l}\right] \psi_{l}=0
$$

\footnotetext{
* Corresponding author. Computational Electronics Group, 3223 Beckman Institute 405 N. Mathews Urbana, IL 61801, USA. E-mail: trell@ceg.uiuc.edu. Tel.: (217)-244-1919. Fax: (217)-244-4333.
} 
coupled with a nonlinear Poisson equation

$$
\nabla(\varepsilon \nabla \phi)=-e\left(-n+p+N_{\mathrm{D}}^{+}-N_{\mathrm{A}}^{-}\right),
$$

where the unknowns are the wavefunctions $\psi_{l}$ belonging to energy levels $E_{l}$, and the electrostatic potential $\phi$. Here, $m^{*}$ is the electron effective mass, $\varepsilon$ the dielectric constant, $n$ and $p$ are the electron and hole concentrations, $N_{\mathrm{D}}^{+}$and $N_{\mathrm{A}}^{-}$the ionized donor and acceptor concentrations, $V_{\mathrm{h}}$ the heterojunction step potential, and finally $V_{\mathrm{xc}}$ the exchange correlation potential in the local density approximation.

The quantum electron density $n$ is obtained from the eigenpairs $\left(E_{l}, \psi_{l}\right)$ of Schrödinger's equation as

where

$$
n=\sum_{l} N_{l}\left|\psi_{l}\right|^{2}
$$

$$
N_{l}=\left(\frac{2 m^{*} k_{\mathrm{B}} T}{\pi^{2} \hbar^{2}}\right)^{1 / 2} \mathcal{F}_{-1 / 2}\left(\frac{E_{\mathrm{F}}-E_{l}}{k_{\mathrm{B}} T}\right)
$$

denotes the occupancy of the $l$-th eigenstate, and $T$ is the Temperature, $k_{\mathrm{B}}$ Boltzmann's constant, $E_{\mathrm{F}}$ the Fermi level, and $\mathcal{F}_{-1 / 2}$ the complete FermiDirac integral of order $-1 / 2$.

We avoid the expense of solving Schrödinger's equation in a very large grid by using the quantum formulation for $n$ (3) only within the region of the quantum wire itself, while in the rest of the simulation domain, where the electron density is small, we replace it by its classical expression. The hole concentration $p$ is very small throughout the quantum wire, but needs to be included into Poisson's equation to ensure a correct description of the band bending in the substrate [1].

\section{SOLUTION BY UNDERRELAXATION}

This system of coupled nonlinear differential equations is usually solved by an iteration between Poisson's and Schrödinger's equation [2, 3]. Since a plain iteration by itself does not converge, it is necessary to underrelax in the electron density $n$ using an adaptively determined relaxation parameter $\omega^{(k)}$. This underrelaxation approach can be briefly outlined as follows:

1. Solve nonlinear Poisson equation using the old electron density $n^{(k-1)}$ to obtain electrostatic potential $\phi^{(k)}$.

2. Solve Schrödinger's equation using $\phi^{(k)}$ and $V_{\mathrm{xc}}\left(n^{(k-1)}\right.$ to obtain a new set of eigenpairs $\left(E_{l}^{(k)}, \psi_{l}^{(k)}\right)$.

3. Calculate an intermediate electron density $n_{\mathrm{int}}^{(k)}$.

4. Choose an appropriate relaxation parameter $\omega^{(k)}$ to obtain new density $n^{(k)}$, and repeat outer iteration until $n$ becomes stationary.

The weakness of this method is the inherent instability of the outer iteration which is controlled by the underrelaxation procedure only. The relaxation parameter $\omega^{(k)}$ is not known in advance and needs to be dynamically readjusted during the course of the iteration. If $\omega^{(k)}$ is too large, the total quantized charge $\int n d x$ oscillates from one iteration step to the other without reaching convergence, while if $\omega^{(k)}$ is too small, too many iteration steps are necessary to achieve convergence.

\section{SOLUTION BY A PREDICTOR- CORRECTOR TYPE APPROACH}

To address this problem we have to modify the underrelaxation algorithm in a way that partially decouples both partial differential equations and damps the oscillations in the total electric charge. We can achieve this goal by incorporating a modified expression for the quantum electron density $\tilde{n}(\phi)$ into Poisson's equation, which approximates the implicit dependency of the quantum electron density $n$ on the electrostatic potential $\phi$ due to Schrödinger's equation.

A suitable expression of this type is provided by quantum mechanical perturbation theory. Using its formalism one can show $[4,5]$, that for a small perturbation of the electrostatic potential $\delta \phi$ the 
occupancies $N_{i}$ within the electron density (3) become $\phi$-dependent as

$$
\begin{gathered}
N_{l}(\delta \phi) \approx \tilde{N}_{l}(\delta \phi) \\
=\left(\frac{2 m * k_{\mathrm{B}} T}{\pi^{2} \hbar^{2}}\right)^{1 / 2} \mathcal{F}_{-1 / 2}\left(\frac{E_{\mathrm{F}}-E_{l}+e \delta \phi}{k_{\mathrm{B}} T}\right) .
\end{gathered}
$$

In our predictor-corrector approach we utilize this result by applying it to arbitrary large changes in $\phi$ :

We start the $k$-th iteration step by solving a modified Poisson equation

$$
\nabla\left(\varepsilon \nabla \phi^{(k)}\right)=e\left[\tilde{n}\left(\phi^{(k)}\right)-p-N_{\mathrm{D}}^{+}+N_{\mathrm{A}}^{-}\right]
$$

where the potential-independent quantum electron density $n$ is replaced by the potential dependent predictor $\tilde{n}$,

$$
\tilde{n}\left(\phi^{(k)}\right)=\sum_{l} \tilde{N}_{l}^{(k-1)}\left(\phi^{(k)}-\phi^{(k-1)}\right)\left|\psi_{l}^{(k-1)}\right|^{2},
$$

with occupancies $\tilde{N}_{l}^{(k-1)}$ defined as in (6) and the superscript $k-1$ indicating the use of $E_{l}^{(k-1)}$. The electrostatic potential $\phi^{(k)}$ obtained from solving Poisson's equation (7) together with the predicted value for the electron density $\tilde{n}\left(\phi^{(k)}\right)$ is then used within Schrödinger's equation (corrector)

$$
\begin{array}{r}
-\frac{\hbar^{2}}{2} \nabla\left(\frac{1}{m^{*}} \nabla \psi_{l}^{(k)}\right)+\left(V_{h}-e \phi^{(k)}\right. \\
\left.\quad+V_{\mathrm{xc}}\left(\tilde{n}^{(k)}\right)-E_{l}^{(k)}\right) \psi_{l}^{(k)}=0
\end{array}
$$

to calculate a corrected update $n^{(k)}$ to the exact electron density

$$
n^{(k)}=\sum_{l} N_{l}^{(k)}\left|\psi_{l}^{(k)}\right|^{2}
$$

It is essential to insert the latest predictor value $\tilde{n}^{(k)}$ into the exchange correlation in Schrödinger's equation (9), since the numerical experiment shows that the use of $n^{(k-1)}$ within $V_{\mathrm{xc}}$ is detrimental to convergence. The use of $\tilde{n}^{(k)}$ also follows the rule that the latest predictor value should enter the corrector.

The numerical experiment also shows, that, unlike in the standard algorithm, convergence is achieved without underrelaxation, so the outer iteration can be reduced to a simple alternation between Poisson's and Schrödinger's equation until the quantum electron density $n$ becomes stationary:

$$
\operatorname{Residual}(n)=\left\|n^{(k)}-n^{(k-1)}\right\|_{2} \leq \varepsilon .
$$

\section{NUMERICAL RESULTS}

We compared the efficiency of both iteration schemes by computing the bound states for the model device shown in Figure 1 [2]. The quantum wire is based on a GaAs-AlGaAs modulation doped structure, and delimited by two metal gates with a distance of $400 \mathrm{~nm}$. The gate voltages were chosen as $V_{\mathrm{G}}=1.3 \mathrm{~V}$ with respect to the substrate for both contacts; additionally we assumed a Schottky barrier of approximately $1 \mathrm{eV}$ between metal and semiconductor material. The Al concentration was set equal to $26 \%$ throughout the $\mathrm{AlGaAs}$ layer, and we also included a fixed surface charge density of $1.6 \times 10^{12} \mathrm{~cm}^{-2}$ at the GaAs-air interface. All calculations were done at a temperature of $T=4.2 \mathrm{~K}$.

Both Schrödinger's equation as well as Poisson's equation are discretized by a box integration finite difference method to take material discontinuities into account. Since the quantum wire covers only a small part of the entire simulation domain, we employ a non-uniform rectangular mesh concentrated around the wire region to minimize computational cost while retaining high accuracy within the region of interest. For the model device discussed above a $99 \times 88$ grid was found to yield satisfactory spatial resolution and short program run-times on HP100 workstations.

After discretization, Schrödinger's equation becomes a sparse eigenvalue problem, which is 


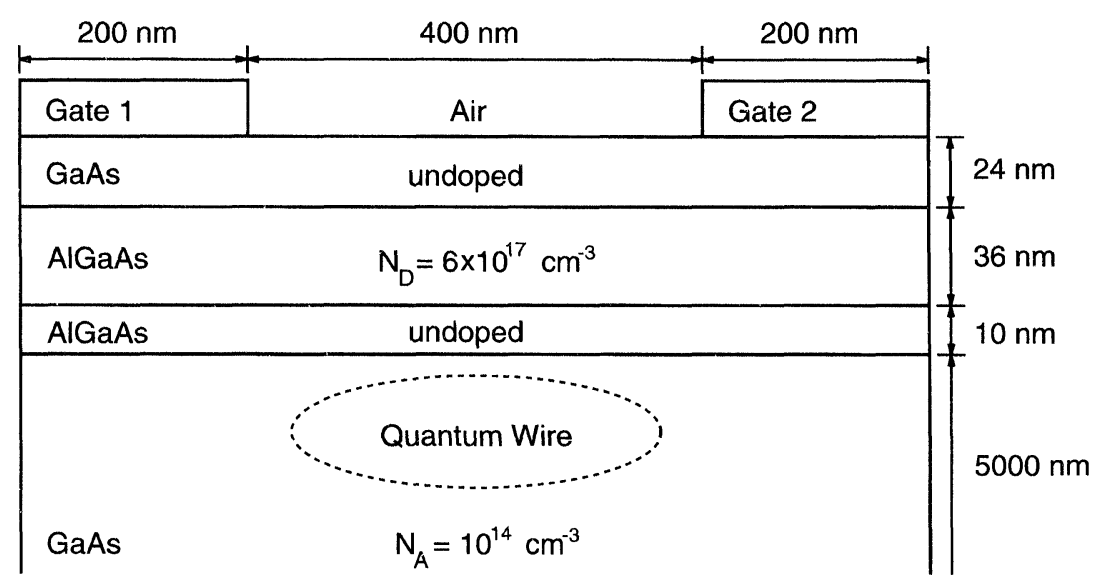

FIGURE 1 The model device structure used in the calculations.

solved by Chebyshev-Arnoldi iteration [6]. This method allows us to compute only the physically relevant lowest-energy states. The nonlinear Poisson equation is solved by Newton-Raphson iteration with inexact line search. The necessary solution of a sparse linear system at each iteration step is accomplished by a version of the preconditioned conjugate gradient method involving redblack reordering. The Fermi-Dirac integrals, which need to be evaluated repeatedly at each grid point, are computed using rational function approximations, which combine speed of execution with high accuracy $[4,7]$.

We compare the convergence speed of the predictor-corrector method with a fast adaptive underrelaxation scheme (an adaptive nonlinear version of the standard Gauss-Seidel algorithm) developed by one of the authors [6], which uses a heuristic method to adjust the relaxation parameter $\omega^{(k)}$. Using the residual in the quantum electron density (11) as measure to quantify convergence we find that our predictor-corrector approach makes the outer iteration stable. In fact, the residual decreases by close to one order of magnitude each step (Fig. 2), which is a 6-fold increase in convergence speed compared to underrelaxation. Additionally we find that the residual decreases uniformly from one step to the next,

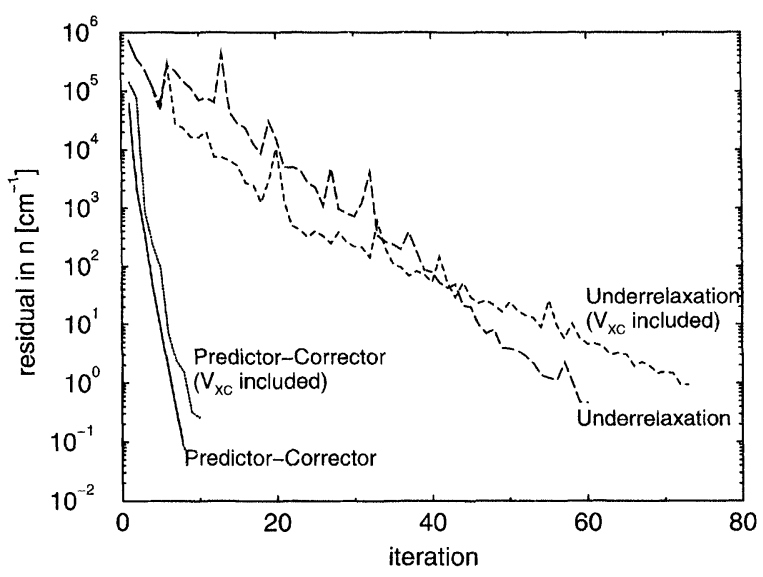

FIGURE 2 Residual of the quantum electron density using a $99 \times 88$ grid.

while for the heuristic underrelaxation algorithm occasional increases are possible.

The inclusion of exchange correlation $V_{\text {xc }}$ results in an additional slowdown of convergence for the underrelaxation scheme and also decreases its stability. However, our predictor-corrector method remains stable and efficient, if the last available predictor value $\tilde{n}^{(k+1)}$ is inserted into $V_{\mathrm{xc}}$ as outlined above.

We verified the rapid uniform convergence of our predictor-corrector method for a wide range of temperatures, gate voltages and geometries, and 
found it in all cases clearly superior to adaptive underrelaxation. This result indicates a fairly wide range of applicability, and considering the ease of implementation of our method we strongly recommend its use even for existing codes.

\section{Acknowledgements}

This work was supported by the National Science Foundation grants ECS 95-09751 and ECD 8943166, Nato Collaborative Grant CRG.950753, and by a graduate fellowship (A.T.) of the Computational Science and Engineering program at the University of Illinois.

\section{References}

[1] Kerkhoven, T., Raschke, M. W. and Ravaioli, U. "Selfconsistent simulation of quantum wires in periodic heterojunction structures", J. Appl. Phys., 74(2), 11991204, July 1993.

[2] Laux, S. E. "Numerical Methods for Calculating SelfConsistent Solutions of Electron States in Narrow Channels", Proceedings of the Fifth International NASECODE Conference, Boole Press, Dublin, Ireland, 1987, pp. 270275.

[3] Kerkhoven, T., Galick, A. T., Ravaioli, U., Ahrends, J. H. and Saad, Y. "Efficient numerical simulation of electron states in quantum wires", J. Appl. Phys., 68(7), 34613469, October 1990.

[4] Trellakis, A., Galick, A. T., Pacelli, A. and Ravaioli, U. "A new iteration scheme for the solution of the twodimensional Schrödinger-Poisson equations in quantum structures", J. Appl. Phys., 81(12), 7880-7884, June 1997.

[5] Pacelli, A. "Self-consistent solution of the Schrödinger equation in quantum wells by implicit iteration", IEEE Trans. Electron Devices, 44(7), 1169-1171, July 1997.

[6] Galick, A. (1993). "Efficient solution of large sparse eigenvalue problems in microelectronic simulation", Ph.D.
Thesis, University of Illinois at Urbana-Champaign, United States.

[7] Trellakis, A., Galick, A. T. and Ravaioli, U. (1997)

"Rational Chebyshev Approximation for the FermiDirac Integral $\mathcal{F}_{-3 / 2}(x)$ ", Solid-State Electron., 41(5) $771-773$.

\section{Authors' Biographies}

Umberto Ravaioli is a professor in the Department of Electrical and Computer Engineering of the University of Illinois at Urbana-Champaign. His fields of professional interest are semiconductor device physics and simulation, numerical methods, and high performance computing.

Andrea Pacelli was a visiting scholar with the Beckman Institute of the University of Illinois at Urbana-Champaign, and he is now a Ph.D. student at the Politecnico di Milano, Italy. His research interests include the physics, modeling and characterization of MOS devices, hot-electron effects and Monte Carlo simulation.

Albert T. Galick is a Postdoctoral Researcher in the Computational Electronics Group in the Beckman Institute of the University of Illinois at Urbana-Champaign. His research focuses on the solution of large scale eigenvalue problems as typical for the simulation of semiconductor devices and dielectric wave-guides.

Alexandros Trellakis is a Ph.D. student at the University of Illinois at Urbana-Champaign. His areas of interest include semiconductor device simulation, simulation of optical cavities, and large-scale simulation of quantum systems. 

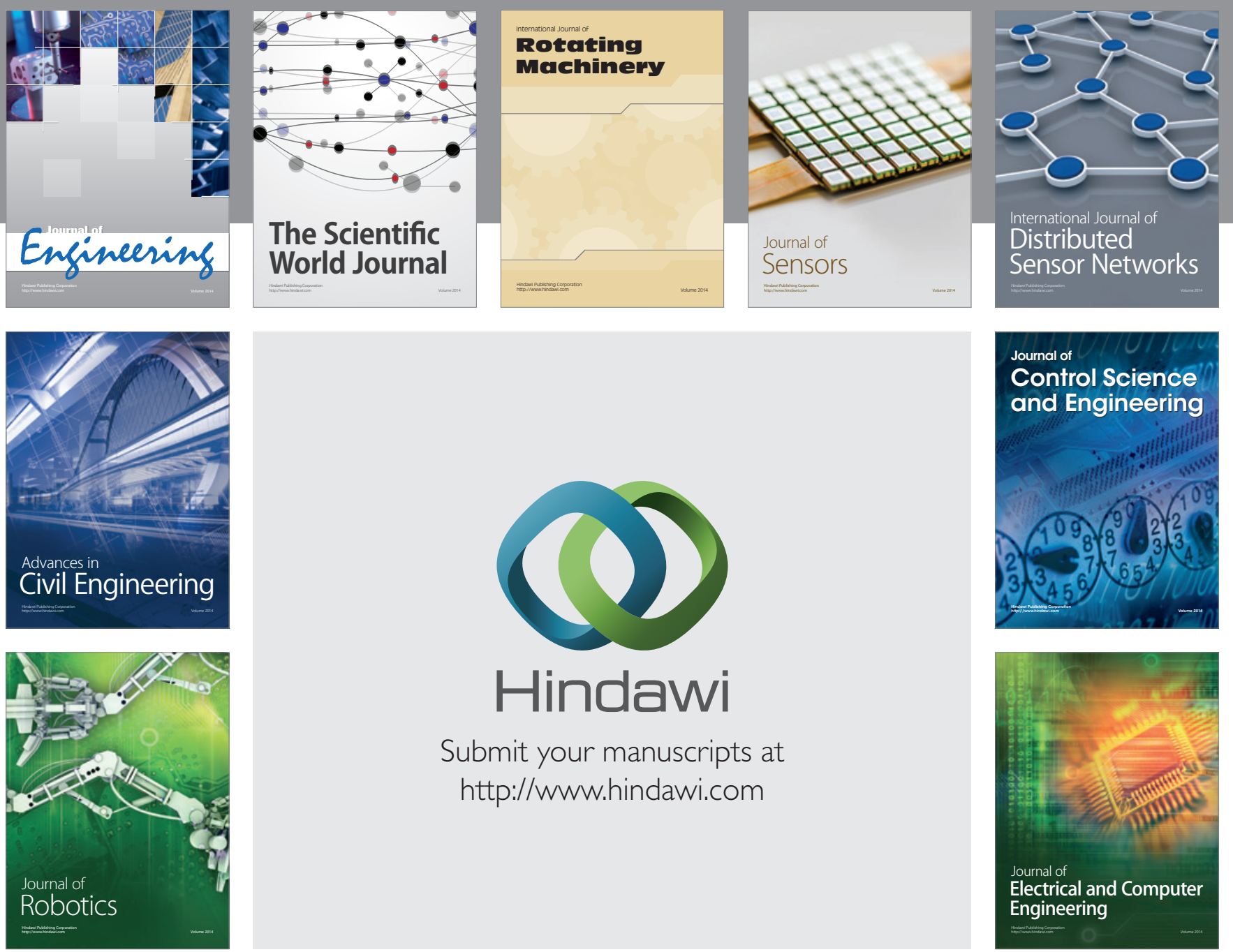

Submit your manuscripts at

http://www.hindawi.com
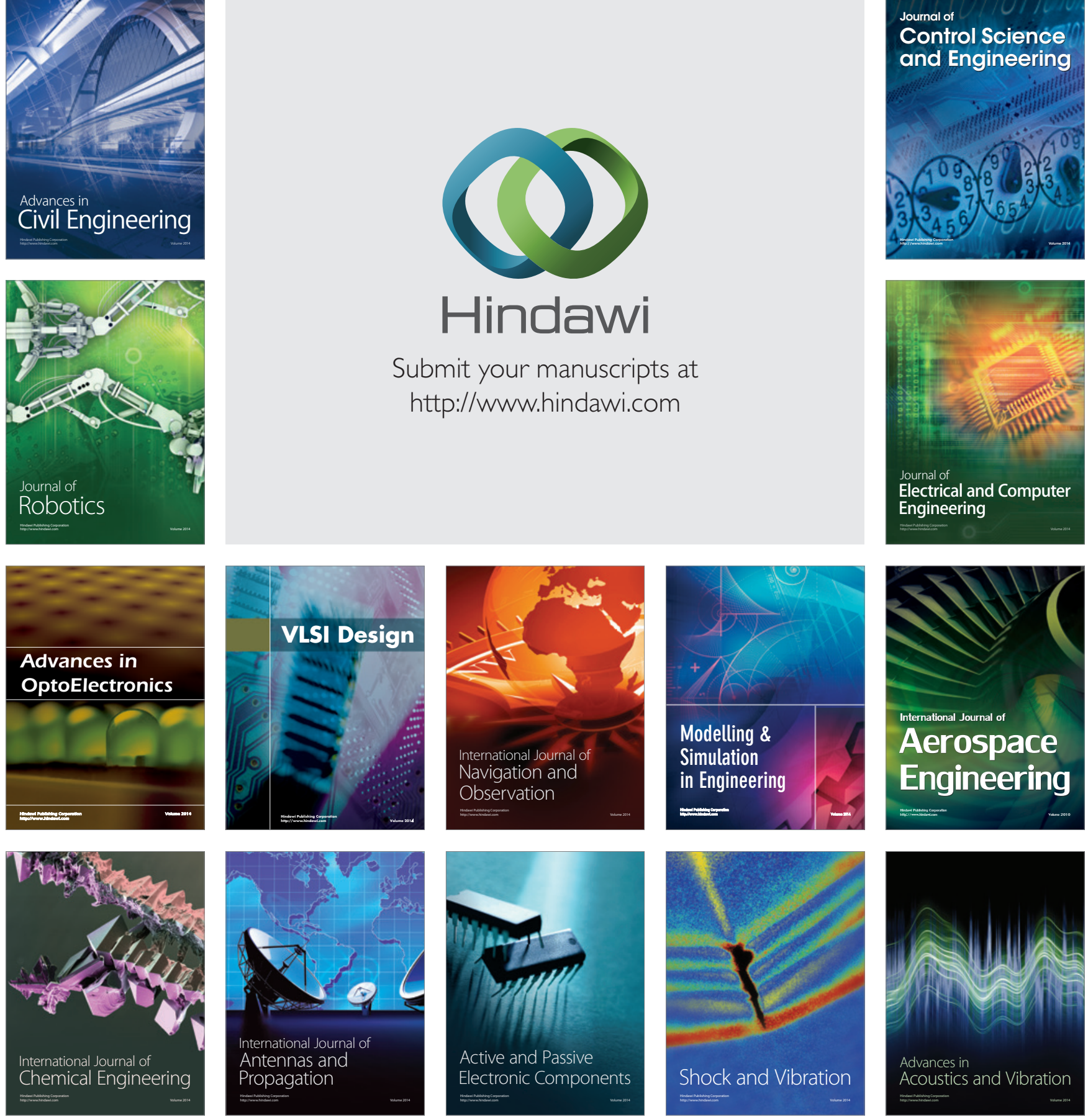